\title{
Anti-Neuronal Antibodies Within the IVlg Preparations: Importance in Clinical Practice
}

\author{
Maria M. Dimitriadou ${ }^{1} \cdot$ Haris Alexopoulos ${ }^{1}$ (D) $\cdot$ Sofia Akrivou $^{1} \cdot$ Eleni Gola ${ }^{1}$ - Marinos C. Dalakas ${ }^{1,2}$ \\ Published online: 31 October 2019 \\ (C) The American Society for Experimental NeuroTherapeutics, Inc. 2019
}

\begin{abstract}
Our study objective was testing for anti-neuronal autoantibodies within commercially available intravenous immunoglobulin (IVIg) preparations. Sixteen samples from 5 different commercially available IVIg preparations were tested with cell-based assays (CBA) and enzyme-linked immunosorbent assay (ELISA) to detect and characterize common neuronal autoantibodies, and with immunohistochemistry on teased fibers from mouse sciatic nerve and on mouse brain sections to screen for nodal and not yet identified neuronal antigens. In 15/16 IVIg preparations, anti-GAD antibodies were detected in titers ranging from 40 to $1507 \mathrm{IU} / \mathrm{mL}$, as typically seen in type 1 diabetes, but not in the range (> $2000 \mathrm{IU} / \mathrm{mL})$ seen in GAD-positive neurological patients. None of the preparations was however positive with anti-GAD CBA. Antibodies to AQP4 were also detected by ELISA in 15/16 IVIg preparations with titers comparable to those seen in AQP4-seropositive NMO patients; with CBA, however, all IVIg samples were AQP4-negative. IVIg preparations contained IgG-anti-MAG antibodies by ELISA at statistically significant higher titers compared to controls. Two of the 16 IVIg samples were positive for human 3-hydroxy-3-methylglutaryl-coenzyme A reductase (HMGCR) antibodies. All IVIg preparations were negative for antibodies to MOG, NMDAR, anti-nodal, and other neuronal-specific proteins. IVIg preparations contain antibodies against GAD and AQP4 in titers comparable to those seen in autoimmune patients when tested by ELISA, but not by CBA or tissue immunohistochemistry, suggesting that the autoantibodies within the IVIg are against linear rather than structural epitopes, as part of the natural antibody immune repertoire. The information is clinically important for diagnosis when testing patients' sera after they have received therapy with IVIg to avoid false interpretation.
\end{abstract}

Keywords Natural autoantibodies $\cdot$ intravenous immunoglobulin $\cdot$ cell-based assay $\cdot$ ELISA $\cdot$ immunohistochemistry

\section{Introduction}

Intravenous immunoglobulin (IVIg) contains high concentration of IgG molecules derived from thousands of healthy blood donors with only traces of detectable IgA and IgM isotypes [1-3]. IVIg is used in autoimmune disorders because of its immunomodulatory effects in inhibiting B-cell functions, hindering complement activation, prevention of T-cell

Maria M. Dimitriadou and Haris Alexopoulos are equal first authors.

Marinos C. Dalakas

mdalakas@med.uoa.gr

1 Neuroimmunology Unit, Department of Pathophysiology, Faculty of Medicine, National and Kapodistrian University of Athens, Athens 11527, Greece

2 Department of Neurology, Thomas Jefferson University, Philadelphia 19107, USA activation pathways, neutralization of pathogenic autoantibodies, and modulation of dendritic cells [4-7]. In neurology, IVIg has been approved for the treatment of Guillain-Barre syndrome (GBS), multifocal motor neuropathy (MMN), and chronic inflammatory demyelinating polyneuropathy (CIDP) and was shown to be effective based on controlled or largescale uncontrolled studies in stiff person syndrome (SPS), myasthenia gravis, dermatomyositis, and certain autoimmune inflammatory myopathies, neuropathies, and central nervous system disorders [7-9].

Because IVIg is derived from healthy donors, it contains substantial amount of natural autoantibodies (NAbs) defined as antibodies present in healthy conditions in the absence of immunizations or infections [3]. The NAbs within the IVIg are exclusively of IgG subclass and they are capable of binding to idiotypes of autoantibodies directed against various self-antigens, thus contributing to restoring immune homeostasis in IVIg-treated patients [10]. Among the NAbs, 
recognition of those directed against neuronal antigenic proteins is important in neurology practice because these antibodies may persist after IVIg administration and have been misinterpreted as pathogenic autoantibodies, often leading to an erroneous diagnosis [11].

The aim of the present study is to investigate the presence of disease-specific neuronal autoantibodies within the IVIg preparations and determine their epitope specificity to assess whether they are directed against structural epitopes with potential pathogenic significance or linear epitopes, as part of the natural antibody immune repertoire. Distinguishing whether detectable antibodies in the patients' sera are derived from the passively infused IVIg or are pathogenic antibodies generated de novo is of clinical importance to avoid diagnostic misinterpretations.

\section{Methods}

The following 16 commercial IVIg products were screened: 10 different lots from HyQvia (Baxalta Innovations $\mathrm{GmbH}$ ), 1 from Privigen (CSL Behring), 3 from Intratect (Biotest AG), 1 from IgVena (Kedrion S.p.A), and 1 from Flebogamma (Grifols S.A.) (Table 1). All brand products used were liquid preparations and no dissolution was needed ensuring the lack or aggregate formation. The following methods were used.

\section{ELISA}

This method was used to test for IgG antibodies against linear epitopes to (a) aquaporin-4 (AQP4) (RSR Limited,
Cardiff, Wales, UK); (b) human 3-hydroxy-3methylglutaryl-coenzyme A reductase (HMGCR) (Inova Diagnostics, Inc., San Diego, CA, USA); (c) myelinassociated glycoprotein (MAG) (BÜHLMANN Laboratories AG, Schönenbuch, Switzerland); and (d) glutamic acid decarboxylase (GAD) (EUROIMMUN Medizinische Labordiagnostika AG, Lübeck, Germany). For AQP4 and GAD detection, none of the IVIg preparations was diluted. No colloids were added as all IVIg preparations tested contain protective colloids. For anti-MAG and HMGCR assays, all IVIg preparations were diluted 1:1000. Sera from 16 healthy people negative for MAG IgG were collected and used as a control group and their mean OD value was compared with the OD of the IVIg preparations.

\section{Anti-GAD Avidity Determined by ELISA}

This method was used to investigate whether polyclonal antibodies within IVIg preparations bind to GAD antigen in a specific manner by testing their avidity using anti-GAD ELISA as follows: one set of duplicate IVIg preparations was incubated with the chaotropic agent sodium thiocyanate $(\mathrm{NaSCN})$ and the other with distilled water [12]. Preliminary experimental data indicated that $1.5 \mathrm{M} \mathrm{NaSCN}$ is the optimal concentration to test avidity. IVIg preparations were then applied undiluted. The relative avidity index (AI), corresponding to the amount of antibodies bound to GAD after incubation with NaSCN to the amount of antibodies bound to GAD without $\mathrm{NaSCN}$, was then calculated and expressed as a percentage.
Table 1 IVIg preparations used in this study

\begin{tabular}{llll}
\hline IVIg brand name & Company & Lot & Concentration \\
\hline HyQvia & Baxalta Innovations GmbH & LE16S281AA & $100 \mathrm{mg} / \mathrm{mL}$ \\
HyQvia & Baxalta Innovations GmbH & LE16S027AB & $100 \mathrm{mg} / \mathrm{mL}$ \\
HyQvia & Baxalta Innovations GmbH & LE16S079AD & $100 \mathrm{mg} / \mathrm{mL}$ \\
HyQvia & Baxalta Innovations GmbH & LE16R324BX & $100 \mathrm{mg} / \mathrm{mL}$ \\
HyQvia & Baxalta Innovations GmbH & LE16R005BC & $100 \mathrm{mg} / \mathrm{mL}$ \\
HyQvia & Baxalta Innovations GmbH & LE16S235AF & $100 \mathrm{mg} / \mathrm{mL}$ \\
HyQvia & Baxalta Innovations GmbH & LE16S104BA & $100 \mathrm{mg} / \mathrm{mL}$ \\
HyQvia & Baxalta Innovations GmbH & LE16S104AJ & $100 \mathrm{mg} / \mathrm{mL}$ \\
HyQvia & Baxalta Innovations GmbH & LE16R066AB & $100 \mathrm{mg} / \mathrm{mL}$ \\
HyQvia & Baxalta Innovations GmbH & LE16S341AJ & $100 \mathrm{mg} / \mathrm{mL}$ \\
Intratect & Biotest AG & B791183 & $50 \mathrm{mg} / \mathrm{mL}$ \\
Intratect & Biotest AG & B791283 & $50 \mathrm{mg} / \mathrm{mL}$ \\
Intratect & Biotest AG & B791473 & $50 \mathrm{mg} / \mathrm{mL}$ \\
Privigen & CSL Behring GmbH & 4327700021 & $100 \mathrm{mg} / \mathrm{mL}$ \\
Ig Vena & Kedrion S.p.A. & 127611 & $50 \mathrm{mg} / \mathrm{mL}$ \\
Flebogamma & Instituto Grifols S.A. & A4GDC00101 & $50 \mathrm{mg} / \mathrm{mL}$ \\
\hline
\end{tabular}




\section{Commercial Cell-Based Assay (CBA) for Anti-AQP4, Anti-MOG, and Anti-NMDAR}

A commercially available method was used to detect for the presence of anti-AQP4, anti-MOG, and anti-NMDAR antibodies according to the manufacturer's instructions (EUROIMMUN Medizinische Labordiagnostika AG, Lübeck, Germany). All IVIg preparations were diluted 1:10 in PBS-Tween. Preparations were viewed using a fluorescent microscope.

\section{In-house CBA for MOG and GAD Proteins}

This method was used to detect antibodies against the whole MOG and GAD proteins, expressed in human embryonic kidney (HEK) cells. The cells were transfected with either a MOG-EGFP or a GAD plasmid with the Xfect reagent (Lipofectamine 2000 Transfection Reagent, Thermo Fisher) followed by a 24 -h incubation with IVIg preparations applied in 1:60 and 1:120 dilutions in 1\% bovine serum albumin (BSA) solution. An anti-GAD commercial polyclonal antibody (1:750, Abcam) or anti-MOG-positive patient serum (1:25) was used as positive control. The wells were then fixed

a

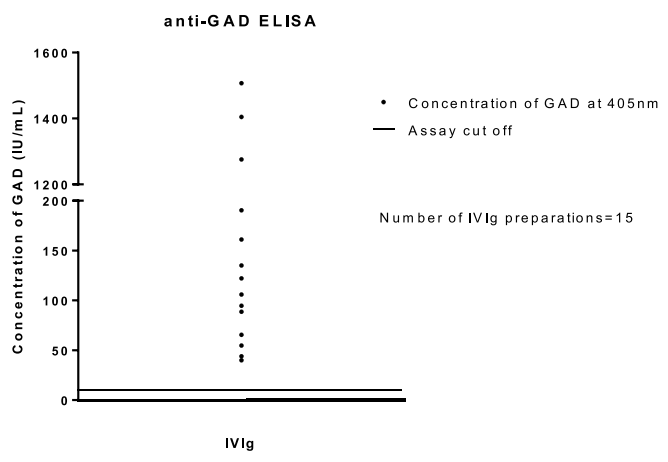

C

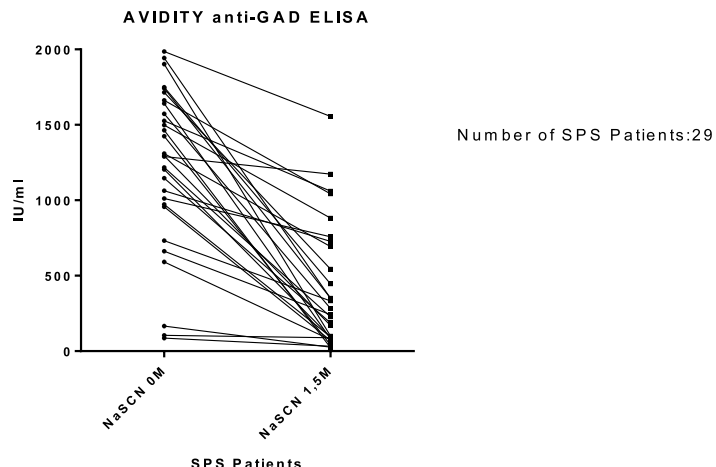

Fig. 1 Anti-GAD antibodies in IVIg preparations. (A) Column graph presenting the results from anti-GAD ELISA. Fifteen out of the $16 \mathrm{IVIg}$ preparations had high titers of GAD antibodies (assay cutoff, positive $\geq$ $10 \mathrm{IU} / \mathrm{mL}$; concentration range, 40,043-1506.96 IU/mL). (B) Immunofluorescent images ( $\times 40$ magnification) using the in-house GAD CBA assay. Positive anti-GAD staining (white arrows) (left), obtained with an anti-GAD-positive commercial antibody; this is in contrast with $4 \%$ paraformaldehyde (PFA) in phosphate-buffered saline (PBS) and permeabilized with $0.1 \%$ Triton in PBS. Following blocking with $10 \%$ normal goat serum (NGS) in PBS, a fluorescent secondary antibody (Alexa Fluor 568) was applied at a 1:750 dilution. DAPI was diluted 1:3000 in PBS to stain nuclei.

\section{Immunohistochemistry}

Teased fibers from mouse sciatic nerve and sagittal mouse brain sections were used to detect the presence of antibodies against unknown brain or peripheral nervous tissue antigens. For the detection of central nervous system (CNS) antigens, sagittal sections of whole adult mouse brain were fixed with $4 \%$ paraformaldehyde (PFA) and permeabilized with the use of the detergent 3-[(3-cholamidopropyl) dimethylammonio]1-propanesulfonate (CHAPS) to allow for the detection of cytoplasmic and nuclear antigenic epitopes. Sections were blocked with $10 \%$ normal goat serum in PBS and all IVIg preparations were applied in a 1:20 dilution in 1\% bovine serum albumin (BSA). The secondary antibody was an Alexa Fluor 488 goat anti-human which was diluted 1:200 in BSA. Cells were counterstained with 4',6-diamidino-2-

b
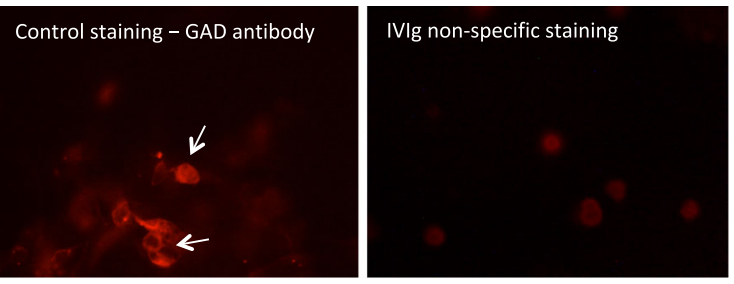

d

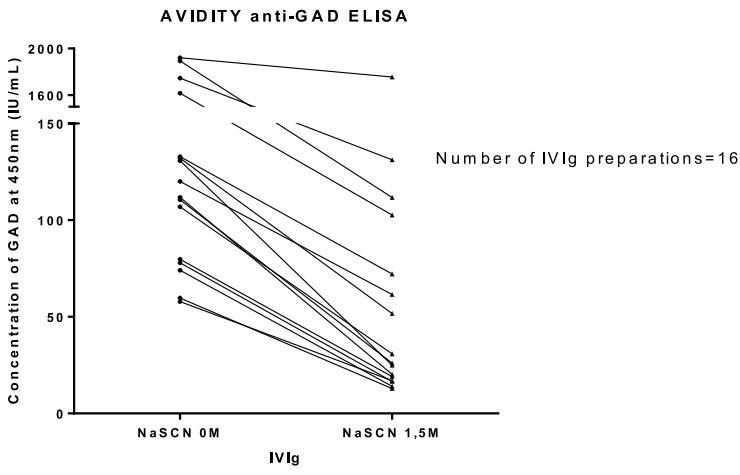

to non-specific staining obtained with IVIg preparation (right). (C, D) Column graph presenting the avidity anti-GAD ELISA data obtained with sera from 29 SPS patients (C) or with IVIg preparations (D). The obtained values of SPS sera had mean avidity index (AI) similar to the values obtained in IVIg preparations. Eleven of the 16 IVIg preparations presented avidity index (AI) values above $20 \%, 3$ presented AI above $18 \%$, and 2 above $5 \%$ 
phenylindole (DAPI) 1:3000 in PBS and preparations were visualized with fluorescent microscopy. For the detection of peripheral nervous system antigens, teased fibers from mouse sciatic nerves were used in a similar protocol [13]. Acetone was used for fixation and $0.5 \%$ Triton-BSA for blocking. All IVIg preparations were diluted 1:50 in 0.5\% Triton blocking solution and incubated overnight at $4{ }^{\circ} \mathrm{C}$. The secondary antibody, which was the same as above, was diluted 1:100 also in $0.5 \%$ Triton blocking solution.

\section{Results}

Fifteen out of 16 IVIg preparations had a high concentration of anti-GAD antibodies as tested with ELISA (assay cutoff, positive $\geq 10 \mathrm{IU} / \mathrm{mL}$; concentration range, 40.043-1506.96 $\mathrm{IU} / \mathrm{mL}$ ); only one IVIg preparation was negative (Fig. 1A).
In contrast, none of the IVIg preparations was tested GADpositive using our in-house CBA method (Fig. 1B). To investigate binding specificity, we calculated avidity index (AI) which showed that 11 out of the 16 IVIg preparations had AI values above 20\%, 3 above 18\%, and 2 above 5\%. This suggests that the titers of anti-GAD antibodies within the IVIg preparations have been reduced significantly after incubation with NaSCN, confirming that the binding of IVIg antibodies to GAD antigen is specific. The obtained AI values are similar to AIs from patients with stiff person's syndrome which harbor high-titer-specific anti-GAD antibodies (Fig. 1C, D).

The AQP4 ELISA testing revealed that all IVIg preparations were positive (assay cutoff, positive $\geq 3.0 \mu / \mathrm{mL}$; concentration range, 3366-39,735 $\mu / \mathrm{mL}$ ), except from a single preparation $(2100 \mu / \mathrm{mL})$. Two other preparations were borderline positive $(3366 \mu / \mathrm{mL}$ and $4093 \mu / \mathrm{mL})$ (Fig. 2A). The range of titers observed in the ELISA testing was comparable

\section{a}

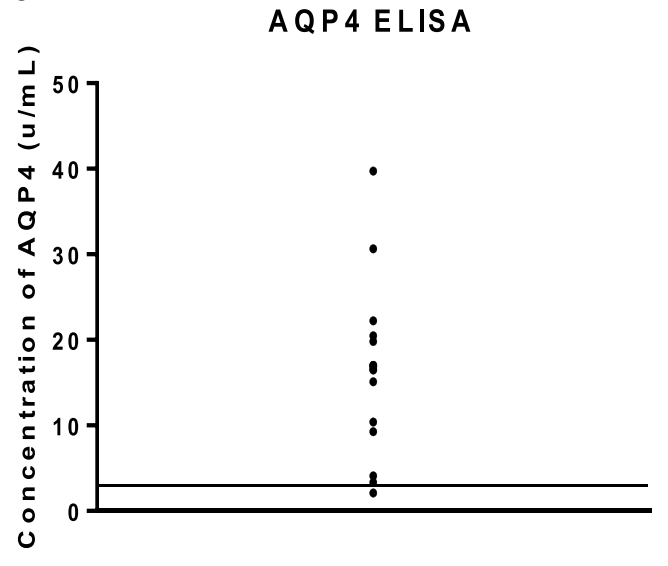

- Concentration of AQP4 at $450 \mathrm{~nm}$

- Assay cut off

Number of IVIg preparations $=16$

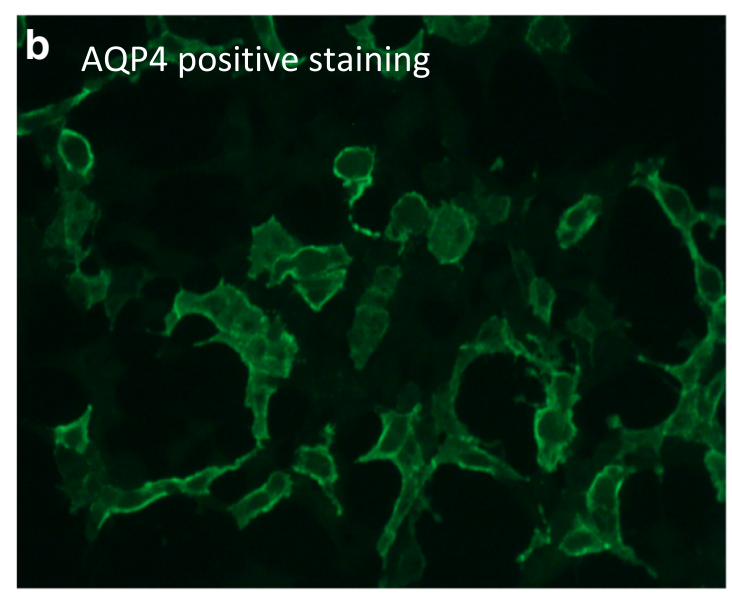

Fig. 2 Anti-AQP4 antibodies in IVIg preparations. (A) Column graph presenting the results from AQP4 ELISA. Fifteen of the 16 IVIg preparations were positive in various concentrations (assay cutoff, positive $\geq$ $3.0 \mu / \mathrm{mL})$. (B, C) Immunofluorescence $(\times 20$ magnification $)$ from an

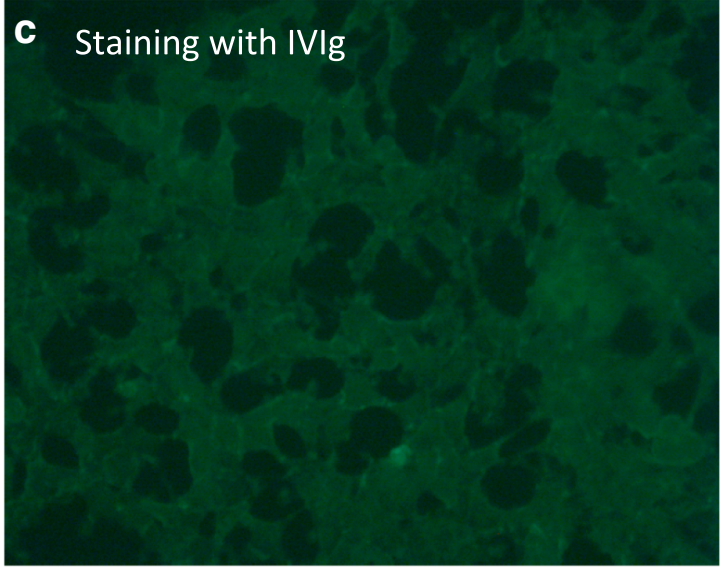

NMOSD-positive control patient shows AQP4-expressing cells using commercial CBA (B); in contrast, no specific staining of AQP4expressing cells was observed with IVIg samples (C) 
to that seen in sera from validated anti-AQP4-positive neuromyelitis optica spectrum patients (data not shown). When the testing was however repeated using the AQP4 CBA, none of the IVIg preparations was positive (Fig. $2 \mathrm{~B}, \mathrm{C}$ ). This is consistent with the observations obtained in tissue sections, in which no specific anti-AQP4 pattern was noted.

The IgG anti-MAG antibodies ELISA testing showed a statistically significant difference between the mean OD value within the 16 IVIg preparations compared with the mean OD value of a group of MAG seronegative patient samples ( $p=$ 0.0002; 99\% confidence interval, 0.08136-0.3446) (dilution, 1:1000) (Fig. 3A). Anti-HMGCR antibodies were detected with ELISA in only 2 IVIg preparations, at low-positive titers (dilution, 1:101; 20,867 standard units and 21,465 standard units) (assay cutoff, positive $\geq 20$ standard units) (Fig. 3B).

Regarding anti-MOG antibodies, none of the tested IVIg preparations was positive (Fig. 4A, B) using both our in-house and commercially available CBA. This was also the case for the NMDAR antibodies using the commercial CBA assay (Fig. 4C, D). For both, MOG and NMDAR antibodies, there is no available validated ELISA method; thus, no additional testing was performed.
None of the IVIg preparations stained brain tissue in a pattern representing neuronal specific staining including specific staining of neuronal or glial cells, e.g., astrocytes. Instead, 15 out of the 16 IVIg preparations stained nuclei in the mouse brain sections (Fig. 5A). It was not investigated further whether this anti-nuclear staining represents any known intracellular epitope. Finally, there was no specific staining on the teased nerve fibers with any of the IVIg preparations (Fig. 5C) denoting the lack of antibodies against nodal antigens such as neurofascin-155, CASPR2, or contactin.

\section{Discussion}

Our data show that IVIg preparations contain several specificities of antibodies observed in the diagnosis of autoimmune neurological diseases, namely against GAD, AQP4, and MAG. Positivity was however only observed with ELISA, a method that allows for the recognition of linear antigenic epitopes and not with cell-based assay that determines structural epitopes. This was particularly evident for the IVIg antibodies
Fig. 3 MAG and HMGCR antibodies in IVIg preparations. (A) Column graph of anti-MAG ELISA. There was a statistically significant difference between the mean value of the 16 IVIg preparations and the mean value of 16 MAG IgG-seronegative controls ( $p=0.0002 ; 99 \%$ confidence interval, 0.08136-0.3446) (dilution, $1: 1000$ ). (B) Column graph of HMGCR ELISA tests. Only 2 of the 16 IVIg samples were lowpositive (dilution, 1:101;20,867 SGU and 21,465 SGU; assay cutoff, positive $\geq 20$ SGU)

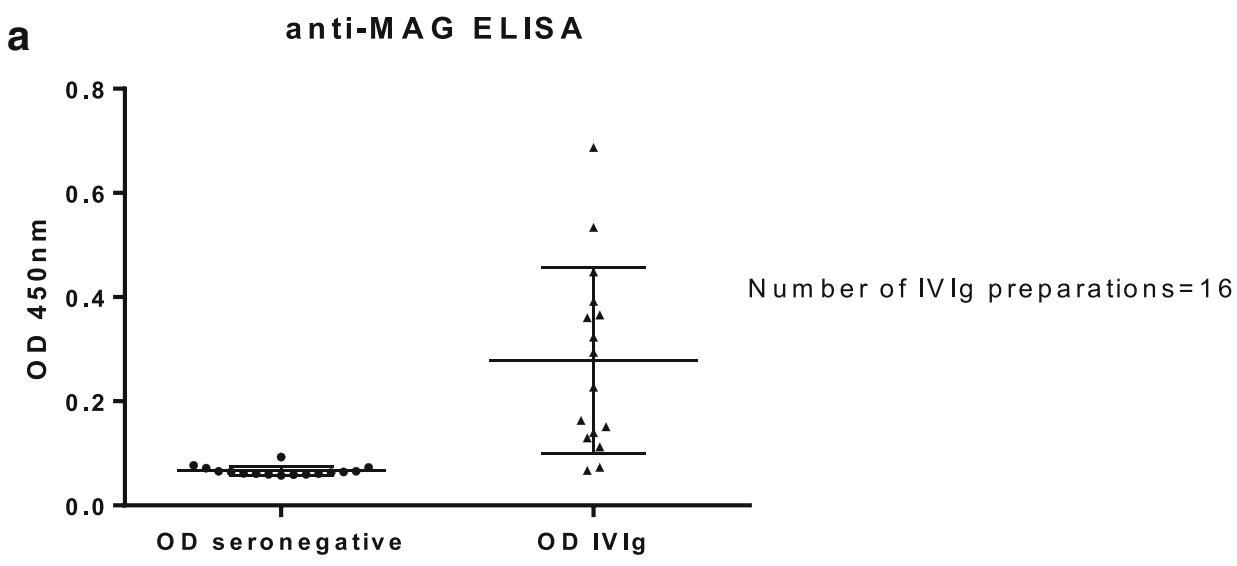

b

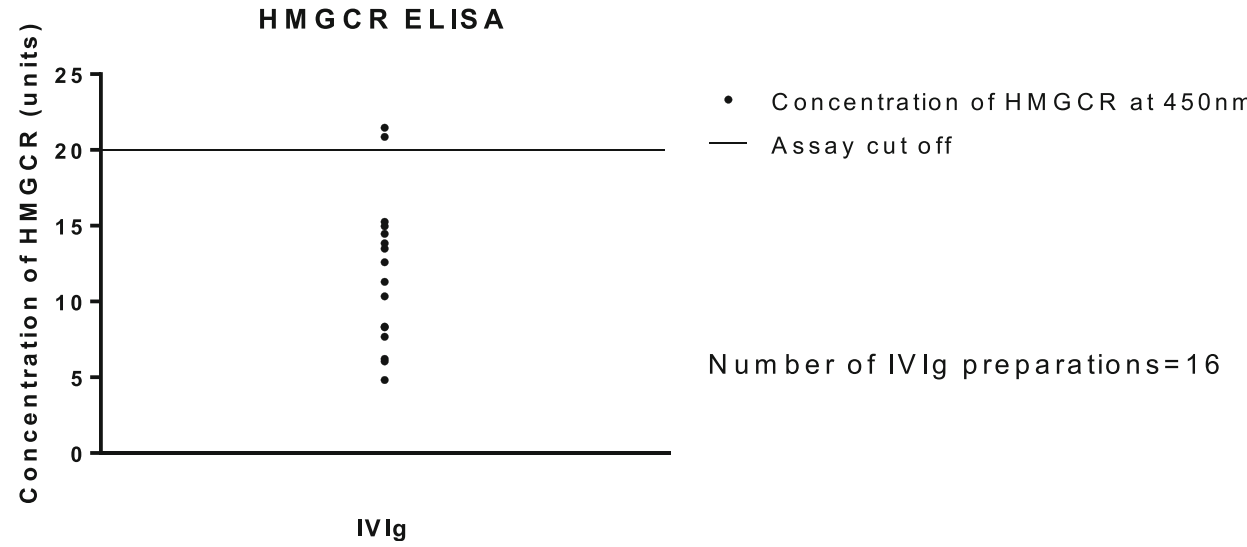



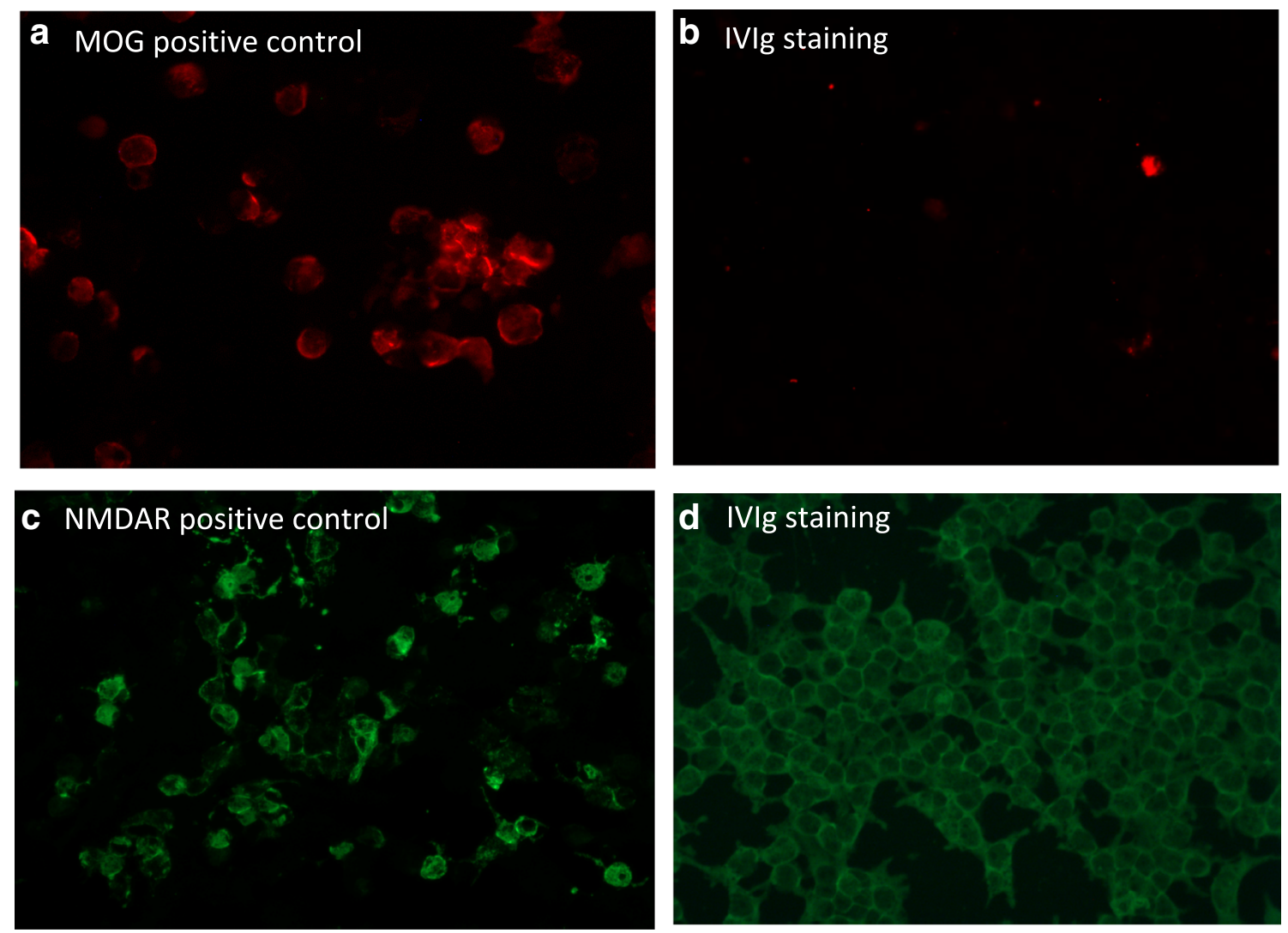

Fig. 4 MOG and NMDAR antibodies in IVIg preparations. (A, B) Immunofluorescent images ( $\times 40$ magnification) show a MOG-positive control serum using in-house MOG CBA (A) compared to non-specific

binding of IVIg (B). (C, D) Immunofluorescent images $(\times 20$ magnification) of an NMDAR-positive control serum using commercial NMDAR CBA (C) compared to non-specific binding with IVIg (D)

against GAD and AQP4 in which a direct comparison between ELISA and CBA was possible.

In reference to GAD, IVIg preparations contain anti-GAD natural antibodies in titers comparable to those seen in patients in type 1 diabetes (TID), but not in patients with stiff person syndrome (SPS), as shown previously with radioimmunoassay [14]. The antibody binding is however specific based on the avidity index, because the anti-GAD IgG within IVIg binds to GAD antigen with strength comparable to antiGAD IgG derived from neurological patients. Whether these antibodies may exert any pathogenic effect is not clear. Although the titers are below those seen in SPS, their presence requires caution when screening for disease-associated GAD antibodies after IVIg infusions. Such results have often created misdiagnoses in a number of patients and only follow-up testing 3 weeks later, when the high titers have been lowered, clarified that they were IVIg-derived (Dalakas MC unpublished observations). Elevated antibody titers in the patients' sera following IVIg treatment have been previously reported for anti-TPO (thyroid peroxidase) and caution was advised not to interpret this as denoting autoimmune thyroiditis [15].

Of interest were the anti-AQP4 antibodies found in IVIg. Their titers within the preparations when evaluated by AQP4 ELISA assay were comparable to those seen in patients with

neuromyelitis optica spectrum disorders (NMOSD). In contrast to NMOSD patients, however, in which the AQP4 ELISA-positive patients are also positive with CBA, in the IVIg, these antibodies were undetectable with CBA suggesting that they recognize only linear epitopes. It should be however acknowledged that because within the IVIg preparations $\mathrm{IgG}$ concentration is very high, epitope density in the ELISA assays is also much higher and the observed positivity may not necessarily represent a physiologically relevant reactivity.

Similar observations were made in a previous study in which clinically relevant AQP4 antibodies were detected within the sera of several patients with autoimmune diseases, such as SLE and even MS, and these antibodies mapped to linear epitopes $[16,17]$. As with the anti-GAD antibodies, however, it is not clear whether the anti-AQP4 antibodies within the IVIg bear any pathogenic potential because an ELISA assay cannot distinguish between natural and pathogenic autoimmunity, hence the need for CBA testing to avoid false-positive results. It is very likely that these antibodies, if physiologically relevant, may represent natural autoantibodies. Potential pathogenicity could be examined by applying the IVIg-derived antibodies in astrocytic cultures in the presence of complement to test for cell death [18]. Because many of these reactivities, like anti-AQP4, anti-GAD, anti- 

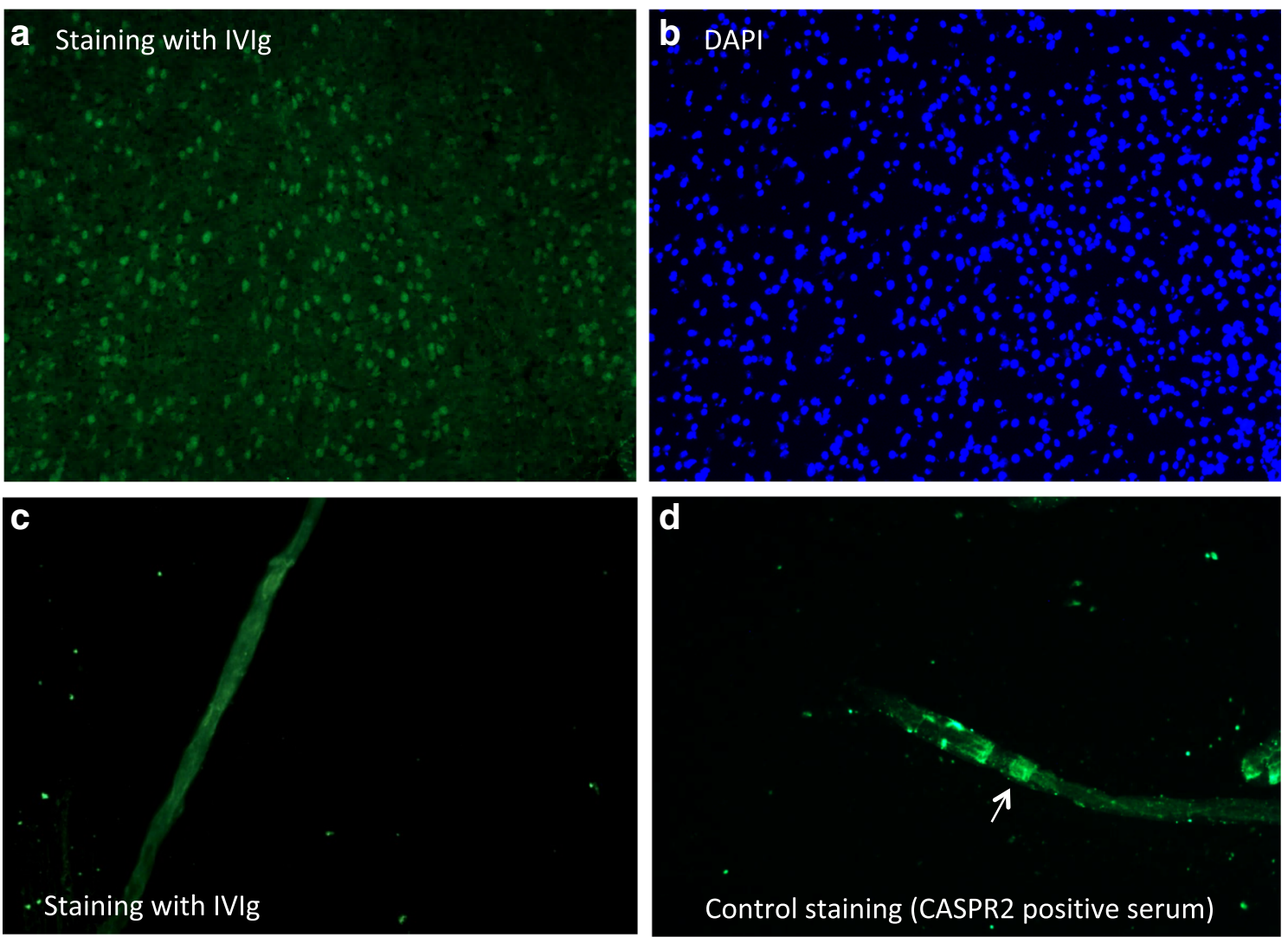

Fig. 5 Tissue staining of IVIg preparations. (A, B) Immunofluorescent images ( $\times 20$ magnification) of anti-nuclear staining of IVIg in a cortical brain area of a mouse sagittal brain section (A). Staining of the same area with 4',6-diamidino-2-phenylindole (DAPI) confirms nuclear staining (B). (C, D) Immunofluorescent images ( $\times 40$ magnification) of a teased

HMGCR, or anti-MAG, even if derived from patients with severe disease, cannot induce pathology in animal passive transfer models [19]; it is highly unlikely that their presence as natural autoantibodies within the IVIg, without any other precipitating factors, can induce any pathology,

Another important message of the study is that IVIg preparations do not contain natural antibodies against NMDAR and MOG. Anti-HMGCR was also rarely present, detected only in 2/16 IVIg samples, supporting that our ELISA findings are specific and not an ELISA artifact, due to Ig concentration. The presence of anti-MAG reactivity in IVIg preparations is also of unclear clinical significance, as patient-derived pathogenic anti-MAG antibodies are of the IgM isotype [20]. The information is however useful as many commercial assays test also for IgG anti-MAG antibodies as part of their panel.

The abundance of anti-linear antibodies in IVIg, as determined by ELISA, was also supported by the finding that no staining was observed in the teased nerve fiber preparations or the brain tissue sections, except of a non-specific nuclear staining. Such anti-nuclear staining resembles the staining seen in the sera of patients with systemic autoimmune nerve fiber from mouse sciatic nerve with IVIg shows non-specific background staining at the node of Ranvier (C); this is in contrast to a positive paranodal staining (white arrow) of anti-CASPR2-positive CIDP serum (positive control) (D)

rheumatic diseases [21], suggesting that IVIg contains antinuclear antibodies in titers comparable to those seen in such patients. Whether the natural autoantibodies within the IVIg account for any of the reported neurological or systemic side effects and laboratory abnormalities after IVIg treatments [22, 23], such as aseptic meningitis, fever, headaches, thrombotic events, fatigue, lymphopenia, anemia, or hyponatremia, if they recognized respective antigens, is unclear although unlikely. It should also be stressed that this point applies to IVIg in general and it is not related to any specific brands used in our testing.

The observed antibodies within the IVIg represent natural autoantibodies, probably unrelated to disease pathobiology; their functional role in physiology however is still unclear and merits further investigation. In healthy populations, natural autoantibodies are of very low concentration but can still be detected following Ig purification. IVIg is a pool of thousands of individuals and accumulation of very low antibody concentrations from single individuals cross the threshold levels of detection. These antibodies are part of the natural autoimmunity repertoire which, in healthy individuals, plays a role in physiological immune regulation. The neurologist 
should be therefore aware when testing patients' sera up to a 4week period after they have received therapy with IVIgconsistent with the IgG's half-life to avoid false interpretation.

Acknowledgments We wish to thank Assist. Prof. E. Kapsogeorgou for technical assistance.

Data Availability All data relevant to this article are presented in the "Results" section. Raw data are available for inspection on request from the corresponding author.

\section{References}

1. Kaveri SV. Intravenous immunoglobulin: exploiting the potential of natural antibodies. Autoimmun Rev. 2012;11(11):792-794. doi: https://doi.org/10.1016/j.autrev.2012.02.006.

2. Kazatchkine MD, Kaveri SV. Immunomodulation of autoimmune and inflammatory diseases with intravenous immune globulin. $\mathrm{N}$ Engl J Med. 2001;345(10):747-755. doi:https://doi.org/10.1056/ NEJMra993360

3. Lacroix-Desmazes S, Kaveri SV, Mouthon L, Ayouba A, Malanchere E, Coutinho A et al. Self-reactive antibodies (natural autoantibodies) in healthy individuals. J Immunol Methods. 1998;216(1-2):117-137.

4. Lunemann JD, Nimmerjahn F, Dalakas MC. Intravenous immunoglobulin in neurology-mode of action and clinical efficacy. Nature reviews Neurology. 2015;11(2):80-89. doi:https://doi.org/10.1038/ nrneurol.2014.253.

5. Dalakas MC. Mechanistic effects of IVIg in neuroinflammatory diseases: conclusions based on clinicopathologic correlations. Journal of clinical immunology. 2014;34 Suppl 1:S120-S126. doi: https://doi.org/10.1007/s10875-014-0024-5.

6. Dalakas MC. Blockade of blocking antibodies in Guillain-Barre syndromes: "unblocking" the mystery of action of intravenous immunoglobulin. Annals of neurology. 2002;51(6):667-669. doi: https://doi.org/10.1002/ana.10259.

7. Dalakas MC. Intravenous immunoglobulin in autoimmune neuromuscular diseases. Jama. 2004;291(19):2367-2375. doi:https://doi. org/10.1001/jama.291.19.2367.

8. Dalakas MC. Intravenous immune globulin therapy for neurologic diseases. Annals of internal medicine. 1997;126(9):721-730.

9. Dalakas MC. Mechanism of action of intravenous immunoglobulin and therapeutic considerations in the treatment of autoimmune neurologic diseases. Neurology. 1998;51(6 Suppl 5):S2-S8. doi:https:// doi.org/10.1212/wnl.51.6_suppl_5.s2.

10. Vani J, Elluru S, Negi VS, Lacroix-Desmazes S, Kazatchkine MD, Bayry J et al. Role of natural antibodies in immune homeostasis: IVIg perspective. Autoimmun Rev. 2008;7(6):440-444. doi:https:// doi.org/10.1016/j.autrev.2008.04.011.

11. de Beer F, Schreurs MW, Foncke EM. False positive autoantibodies to glutamic acid decarboxylase in opsoclonus-myoclonus-ataxia syndrome after intravenous treatment with immunoglobulin. Clin
Neurol Neurosurg. 2009;111(7):643-644. doi:https://doi.org/10. 1016/j.clineuro.2009.03.010.

12. Suwannalai P, Scherer HU, van der Woude D, Ioan-Facsinay A, Jolvan der Zijde CM, van Tol MJ et al. Anti-citrullinated protein antibodies have a low avidity compared with antibodies against recall antigens. Ann Rheum Dis. 2011;70(2):373-379. doi:https://doi.org/ 10.1136/ard.2010.135509.

13. Stathopoulos P, Alexopoulos H, Dalakas MC. Autoimmune antigenic targets at the node of Ranvier in demyelinating disorders. Nature reviews Neurology. 2015;11(3):143-156. doi:https://doi. org/10.1038/nrneurol.2014.260.

14. Smith TD, Cunningham-Rundles C. Detection of anti-glutamic acid decarboxylase antibodies in immunoglobulin products. J Allergy Clin Immunol Pract. 2018;6(1):260-261. doi:https://doi.org/10. 1016/j.jaip.2017.04.042.

15. Abbott JK, Church JA. In vivo assessment of clinically relevant autoantibodies in intravenous immunoglobulin preparations. Pediatric Allergy, immunology and pulmonology. 2010;23(2): 121-123. doi:https://doi.org/10.1089/ped.2010.0009.

16. Kampylafka EI, Routsias JG, Alexopoulos H, Dalakas MC, Moutsopoulos HM, Tzioufas AG. Fine specificity of antibodies against AQP4: epitope mapping reveals intracellular epitopes. J Autoimmun. 2011;36(3-4):221-227. doi:https://doi.org/10.1016/j. jaut.2011.01.004.

17. Alexopoulos H, Kampylafka EI, Chatzi I, Travasarou M, Karageorgiou KE, Dalakas MC et al. Reactivity to AQP4 epitopes in relapsing-remitting multiple sclerosis. Journal of neuroimmunology. 2013;260(1-2):117-120. doi:https://doi.org/ 10.1016/j.jneuroim.2013.04.017.

18. Alexopoulos H, Kampylafka EI, Fouka P, Tatouli I, Akrivou S, Politis PK et al. Anti-aquaporin-4 autoantibodies in systemic lupus erythematosus persist for years and induce astrocytic cytotoxicity but not CNS disease. Journal of neuroimmunology. 2015;289:8-11. doi:https://doi.org/10.1016/j.jneuroim.2015.10.007.

19. Bradl M, Lassmann H. Experimental models of neuromyelitis optica. Brain pathology (Zurich, Switzerland). 2014;24(1):74-82. doi:https://doi.org/10.1111/bpa.12098.

20. Dalakas MC. Advances in the diagnosis, immunopathogenesis and therapies of IgM-anti-MAG antibody-mediated neuropathies. Ther Adv Neurol Disord. 2018;11:1756285617746640. doi:https://doi. org/10.1177/1756285617746640.

21. Nishimura S, Nishiya K, Hisakawa N, Chikazawa H, Ookubo S, Nakatani $\mathrm{K}$ et al. Positivity for antinuclear antibody in patients with advanced rheumatoid arthritis. Acta Med Okayama. 1996;50(5): 261-265. doi:https://doi.org/10.18926/AMO/30501.

22. Guo Y, Tian X, Wang X, Xiao Z. Adverse Effects of Immunoglobulin Therapy. Front Immunol. 2018;9:1299. doi: https://doi.org/10.3389/fimmu.2018.01299.

23. Koffman BM, Dalakas MC. Effect of high-dose intravenous immunoglobulin on serum chemistry, hematology, and lymphocyte subpopulations: assessments based on controlled treatment trials in patients with neurological diseases. Muscle \& nerve. 1997;20(9): $1102-1107$.

Publisher's Note Springer Nature remains neutral with regard to jurisdictional claims in published maps and institutional affiliations. 